\title{
Melatonin Attenuates Noise Stress-induced Gastrointestinal Motility Disorder and Gastric Stress Ulcer: Role of Gastrointestinal Hormones and Oxidative Stress in Rats
}

\author{
Lei Zhang, ${ }^{1,2,3} \mathrm{Ji} \mathrm{T}$ Gong, ${ }^{4}$ Hu Q Zhang, ${ }^{5}$ Quan H Song, ${ }^{2}$ Guang H Xu, ${ }^{3}$ Lei Cai, ${ }^{3}$ Xiao D Tang, ${ }^{2}$ Hai F Zhang, ${ }^{1}$ Fang-E Liu, ${ }^{1 *}$ \\ Zhan S Jia ${ }^{6 *}$ and Hong W Zhang ${ }^{3 *}$ \\ ${ }^{1}$ Experiment Teaching Center of Basic Medicine, The Fourth Military Medical University, Xi'an, Shan Xi province, China; ${ }^{2}$ Department of General \\ Surgery, No. 406 hospital, Da Lian, Liao Ning Province, China; ${ }^{3}$ State Key Laboratory of Cancer Biology, Xijing Hospital of Digestive Diseases, \\ The Fourth Military Medical University, Xi' an, Shan Xi Province, China; ${ }^{4}$ Department of Otorhinolaryngology, Baoji Hospital of Chinese \\ Medicine, Shan Xi Province, China; ${ }^{5}$ The Key Laboratory of Biomedical Information Engineering of Ministry of Education, School of Life Science \\ and Technology, Xi' an Jiaotong University, Xi' an, Shan Xi Province, China; and ${ }^{6}$ Department of Infectious Diseases, Tangdu Hospital, the Fourth \\ Military Medical University, Xi' an, China
}

\section{Background/Aims}

There are increasing evidences for gastrointestinal motility disorder (GIMD) and gastric stress ulcer induced by noise stress. The present study was to investigate the reversed effect of melatonin on GIMD and gastric stress ulcer induced by noise stress and potential mechanism.

\section{Methods}

Noise stress was induced on rats, and melatonin $(15 \mathrm{mg} / \mathrm{kg}$ ) was administered to rats by intraperitoneal injection. Differences were assessed in gastric residual rate (GRR), small intestine propulsion rate (SPR), Guth injury score, cortisol, gastrointestinal hormones (calcitonin-gene-related peptide and motilin) and oxidative stress markers (superoxide dismutase and malondialde

Received: October 9, 2014 Revised: November 5, 2014 Accepted: November 5, 2014

(c) This is an Open Access article distributed under the terms of the Creative Commons Attribution Non-Commercial License (http://creativecommons. org/licenses/by-nc/3.0) which permits unrestricted non-commercial use, distribution, and reproduction in any medium, provided the original work is properly cited.

*Correspondence: Fang-E Liu, Zhan S Jia, and Hong W Zhang are equally responsible for this study.

Fang-E Liu, MD, PhD

Experiment Teaching Center of Basic Medicine, School of Basic Medicine, Fourth Military Medical University, Xi’an, 710033 Shan Xi Province, China

Tel: +86-29-8477-4518, Fax: +86-29-8477-4764, E-mail: liufange@fmmu.edu.cn

Zhan S Jia, MD, PhD

Department of Infectious Diseases, Tangdu Hospital, the Fourth Military Medical University, Xi'an, 710038 Shan Xi Province, China Tel: +86-29-8477-7652, Fax: +86-29-8353-7377, E-mail: jiazsh@fmmu.edu.cn

Hong W Zhang, MD, PhD

State Key Laboratory of Cancer Biology, Xijing Hospital of Digestive Diseases, The Fourth Military Medical University, Xi'an,

710033 Shan Xi Province, China

Tel; +86-29-8477-3974, Fax: +86-29-8253-9041; E-mail: zhanghw@fmmu.edu.cn

Lei Zhang and Ji T Gong contributed equally to this article.

Financial support: None.

Conflicts of interest: None.

Author contributions: Lei Zhang and Ji T Gong contributed to study concept and design, performed the research, analysis, and interpretation of data, and wrote the manuscript; Hu Q Zhang contributed to performed the research and critical revision of the manuscript; Quan H Song, Guang H Xu, Lei Cai, and Xiao D Tang contributed to data acquisition; Hai F Zhang contributed to analysis and interpretation, and drafting of the manuscript; Fang-E Liu, Zhan S Jia, and Hong W Zhang contributed to study concept and design, analysis and interpretation of data, drafting of manuscript, critical revision of the data for important intellectual content. No competing interests declared. 
hyde) in blood plasma as well as gastric mucosa homogenate with or without melatonin. The pathological examination of gastric mucosa was also performed.

\section{Results}

The GRR and SPR were improved by noise stress compared with control $(P<0.05)$. The pathological examination and Guth injury score revealed gastric stress ulcer. Moreover, the levels of cortisol, motilin and malondialdehyde in blood plasma and malondialdehyde in gastric mucosa homogenate were increased by noise stress $(P<0.05)$. CGRP and superoxide dismutase activity in both of blood plasma and gastric mucosa homogenate were significantly decreased $(P<0.05)$. Furthermore, melatonin reversed changes in GRR, SPR, pathological examination, Guth injury score, cortisol, motilin, CGRP, superoxide dismutase activity and malondialdehyde $(P<0.05)$.

\section{Conclusions}

Melatonin is effective in reversing the GIMD and gastric stress ulcer induced by noise stress. The underlying mechanism may be involved in oxidative stress and gastrointestinal hormones.

(J Neurogastroenterol Motil 2015;21:189-199)

\section{Key Words}

Gastrointestinal hormones; Gastrointestinal motility; Melatonin, Oxidative stress

\section{Introduction}

Stress is a state of threatened homeostasis provoked by psychological, physiological or environmental stressors. ${ }^{1}$ It activates the hypothalamic pituitary adrenal (HPA) axis and the sympathetic nervous system resulting in a physiological change. ${ }^{2}$ Noise stress has been linked to a variety of health issues including cardiovascular diseases, ${ }^{3}$ mental illness, ${ }^{4}$ sleep disorder ${ }^{5}$ and hypertension. ${ }^{6}$ Furthermore, gastrointestinal motility disorder (GIMD), gastritis and peptic ulcers ${ }^{7,8}$ have been noticed in populations who are exposed to high levels of noise. However, little is known about prevention and treatment of noise on GIMD and gastric stress ulcer.

Stress would induce activation of HPA axis that leads to the excessive production of free radicals. Generation of free radicals is an integral feature of normal cellular functions, on the other hand, excessive generation and/or inadequate removal of free radical results in destructive and irreversible damage to cells. ${ }^{9}$ Corticotrophin-releasing hormone is released during stress and stimulates the release of adrenocorticotropic hormone. ${ }^{10}$ It is well established that activation of HPA axis disorganizes gastrointestinal (GI) hormones and up regulates oxidative stress. ${ }^{11}$ GI hormones and oxidative stress may play a critical role in the process of GIMD and gastric stress ulcer induced by noise stress.

Various evidences based on studies conducted in rodents that melatonin (N-acetyl-5-methoxytryptamine, MT), a pineal gland neurohormone, is a neuroendocrine transducer of environmental information with a key role in the circadian organization of vertebrates. ${ }^{12} \mathrm{MT}$ is best known for its antioxidant activities and free radical scavenging ability. ${ }^{13,14}$ The anti-oxidative effect of MT is attributed to both its free radical scavenging activity and the reducing activation of enzymes on the production of reactive oxygen species. ${ }^{15,16}$ Moreover, treatment of pharmacologically effective does of MT during the day to rodents, reported to induce sedation, hypothermia and suppression of HPA axis. ${ }^{17}$

Enterochromaffin cells of the GI tract are the main source of extra pineal MT and substantially contribute to the peripheral blood concentration of MT. ${ }^{18,19}$ While MT acts mostly as an endocrine substance, extra pineal-derived MT functions not only as endocrine, but also as autocrine or paracrine substance and regulates many GI functions such as water and ion transport, proliferation of epithelium, secretion of acid, immune system and motility. ${ }^{19-21}$ Thus, small does of MT intraperitoneal injecting accelerates intestinal transit and gastric emptying in rats besides high does delays the latter action. ${ }^{12}$ But the mechanism underlying MT on GIMD and gastric stress ulcer have not been elucidated.

Considering these data, the aims of the present study were to investigate the effect of MT on noise stress-induced GIMD and gastric stress ulcer in rats. The possible mechanisms were focused on the HPA axis, GI hormones and oxidative stress. 


\section{Materials and Methods}

\section{Animal Preparation}

Animal care was in accordance with the Principles of Medical Laboratory Animal Care issued by the National Ministry of Health. All experimental procedures were performed under the guidelines of the National Ordinances on Experimental animals for the ethical use of animals. The present study was approved from IRB of the Fourth Military Medical University. Adult male SD rats $(n=40,220 \pm 20 \mathrm{~g})$ obtained from the Laboratory Animal Center of the Fourth Military Medical University were used in this study. They were housed 4 rats to a plexiglas cage in a climate-controlled room $\left(20-22^{\circ} \mathrm{C}\right)$ on a $12 / 12$ hour light/dark schedule (lights on at $7 \mathrm{AM}$ ) with a free access to food and water. All rats were allowed to acclimate for 1 week before the experiment.

\section{Drugs Used}

MT (Sigma-Aldrich, St. Louis, MO, USA), superoxide dismutase activity and malondialdehyde kit (Nanjing Jian-Cheng Biotechnology Research, Nanjing, China), calcitonin gene-related peptide (CGRP) and motilin kit (Military 301 Hospital Immunity Technique Research, Beijing, China).

\section{Study Protocol}

Forty rats were divided into 5 groups including 1 control group, 2 stressed groups ( 1 and 2 ) and 2 experimental groups (1 and 2) with 8 rats per group. Rats in stressed groups and experimental groups were given to noise stress $(120 \mathrm{~dB}) 8$ hours everyday: stressed group 1 and experimental group 1 for 1 day; while stressed group 2 and experimental group 2 for 3 days. The rats were fast for 12 hours before the last noise stress. MT was dissolved in a small amount of ethanol and diluted with saline to give a final concentration of ethanol less than $5 \%$. Rats in experimental groups 1 and 2 were given to intraperitoneal injection of MT at a dose of $15 \mathrm{mg} \cdot \mathrm{kg}^{-1}$.day ${ }^{-1}$ (body weight) 30 minutes before the noise stress. The MT does used in this study was chosen on the basis of our previously published experiment. ${ }^{16}$ The other groups of rats received the same volume of saline intraperitoneal injection with coordinate content of ethanol at the same time. All experiments were carried out between $8 \mathrm{AM}$ and $5 \mathrm{PM}$.

\section{Establishment of the Noise-stress Model}

The noise-stress model used in the study was modified from that of $\mathrm{Mu}$ et al. ${ }^{11}$ The rats in stressed groups and experimental groups were confined in an isolated room. Firing noise of submachine guns was recorded on a tape and introduced as an inducing factor, which was played to the rats through a loudspeaker at a distance of $20-30 \mathrm{~cm}$. The intensity of firing noise was measured by a precision pulse counter and a frequency-spectrum analyzer as $120 \mathrm{~dB}(\mathrm{~A})$ with a frequency of $0.25-4.00 \mathrm{kHz}$.

\section{Measurement of Gastric Residual Rate and Small Intestine Peristalsis Rate}

After the last noise stress, rats in each group were given to 2.5 $\mathrm{mL}$ carbon powder suspension which was made of $5 \%$ carbon powder and $10 \%$ arabic gum in distilled water ${ }^{22}$ by intragastric administration. And 20 minutes later, all rats were narcotized by intraperitoneal injecting napental. The abdomen was opened and the stomach was rapidly clamped above the lower esophageal sphincter and beneath the pylorus in order to prevent further passage of carbon powder suspension. The protocol from anaesthesia to abdominal anatomy was taken within one minute by experienced hands. The GI tract from proventriculus to terminal ileum were separated and gently stretched. The stomach was wiped dry and weighted (weight 1, G1), and then it was cut open along the arcus major ventriculi. After the gastric contents were washed out, the stomach was weighted again (weight2, G2). The gastric residual rate (GRR) was calculated from the equation: GRR = [(G1-G2)/weight of $2.5 \mathrm{~mL}$ carbon powder suspension] $\times$ $100 \%$.

The distances from sphincter pylori to the end of carbon powder suspension (distance 1, D1) and to ileocecal junction (distance 2, D2) were measured respectively. The small bowel peristalsis rate $(\mathrm{SPR})$ was calculated from the equation ${ }^{23}$ :

$$
\mathrm{SPR}=(\mathrm{D} 1 / \mathrm{D} 2) \times 100 \% \text {. }
$$

\section{Measurements of Gastric Mucosa Damage}

The gastric mucosa damage was scored according to the Guth method $^{24}$ : the length (L) of a gastric mucosa damage area $<1 \mathrm{~mm}$ was scored as 1 point; $1 \mathrm{~mm} \leq \mathrm{L}<2 \mathrm{~mm}, 2$ points; 2 $\mathrm{mm} \leq \mathrm{L}<3 \mathrm{~mm}, 3$ points; $3 \mathrm{~mm} \leq \mathrm{L}<4 \mathrm{~mm}, 4$ points; and $\mathrm{L} \geq 4 \mathrm{~mm}$, scored segmentally. The total score summed over the whole stomach was used as the ulcer index.

\section{Pathological Examination of Gastric mucosa}

Small pieces of ulcerative gastric mucous membrane were taken and fixed in formalin and kept in $4^{\circ} \mathrm{C}$ for subsequent pathological examination. 


\section{Evaluation of Cortical, Oxidative Stress and Gastrointestinal Hormone in Blood Plasma}

Two $2 \mathrm{~mL}$ blood samples were taken by thoracotomy from heart: one was mixed well by centrifugation at 3,000 rpm/min for 10 minutes and kept at $4^{\circ} \mathrm{C}$, which was ready for detecting superoxide dismutase activity and malondialdehyde level; the other was added with EDTA and retardant peptidase for separation of plasma and kept at $-20^{\circ} \mathrm{C}$ in a refrigerator, which was ready for de-

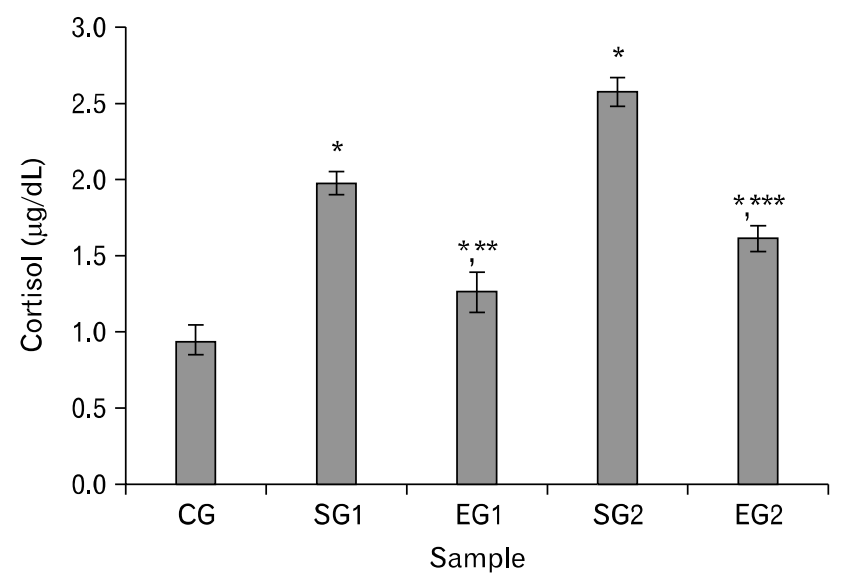

Figure 1. Effect of noise stress on cortisol of blood plasma in rats and the reverse effect of melatonin. Results are shown as mean \pm SEM. For $\mathrm{n}=8,{ }^{*} P<0.05$, significantly different from control group (CG) rats; ${ }^{* *} P<0.05$, significantly different from stressed group 1 (SG1) rats; and ${ }^{* * *} P<0.05$, significantly different from SG2 rats. One-way ANOVA and Fisher's least significant difference tests were used. tecting cortisol, CGRP and motiln.

\section{Detection of Oxidative Stress in Gastric Mucosa}

Gastric mucosa was homogenated to the concentration of 100 $\mathrm{g} / \mathrm{L}$ in ice-cold physiological saline by a homogenizer, and then well mixed with hydroextractor at 3,000 rpm/min for 10 minutes. The supernatant fluid was harvested and stored in $-4^{\circ} \mathrm{C}$ for the detection of superoxide dismutase activity and motilin level.

\section{Statistical Methods}

All data were calculated as mean $\pm \mathrm{SD}$, with $\mathrm{n}$ equal to the number of involved rats. Differences among means of 5 groups were tested with one-way ANOVA. For post hoc testing, Fisher's least significant difference was used in multiple comparisons. A $P$-value $<0.05$ was considered statistically significant. All analyses were performed with SPSS (version 17.0 for Windows, SPSS Inc., Chicago, IL, USA).

\section{Results}

Cortisol of stressed group rats was obviously higher than that of control group or experimental group rats $(P<0.01$, Fig. 1) and it was higher in experimental group rats than in control group rats $(P<0.01$, Fig. 1$)$.

As shown in Figure 2 noise stress significantly enhanced GRR (Fig. 2A) and SPR (Fig. 2B) in stressed group rats. The noise stress effect on the stressed group 2 rats was more apparent
A

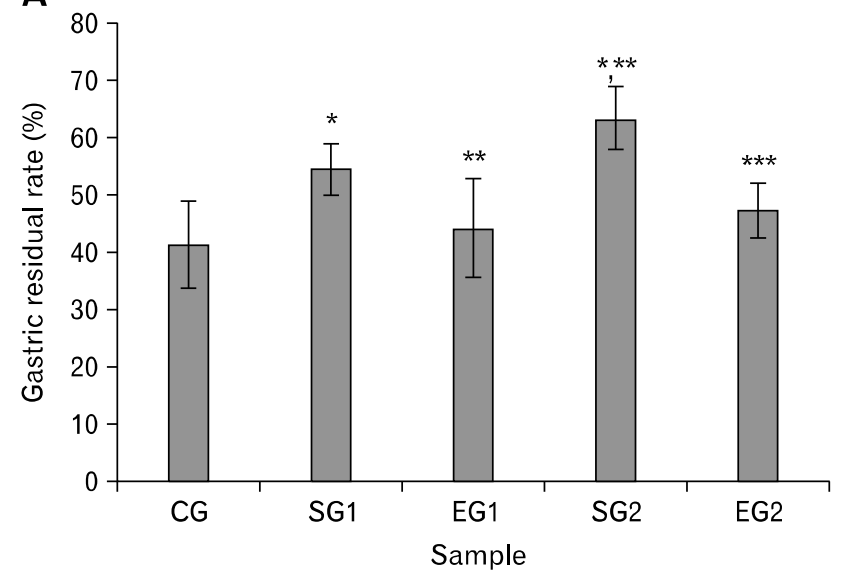

B

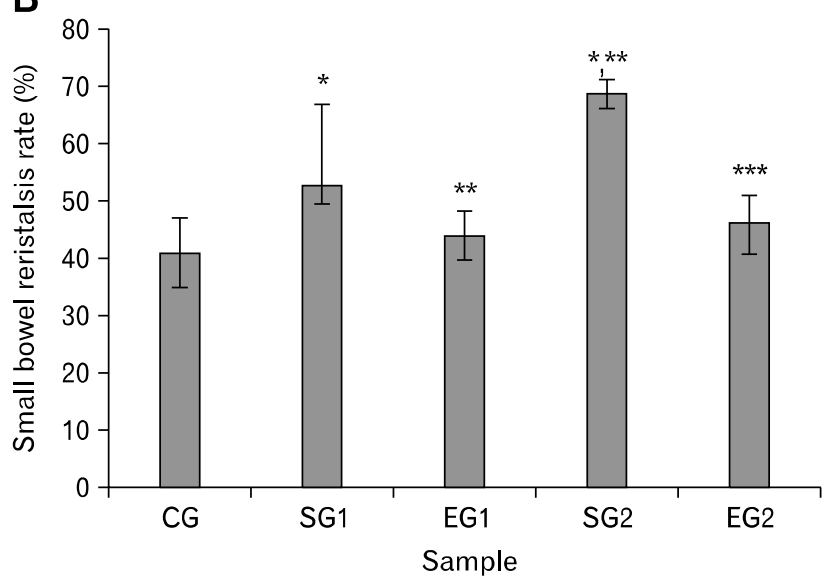

Figure 2. Effect of noise stress on gastric residual rate (A) and small bowel peristalsis rate (B) in rats and the reverse effect of melatonin. Results are shown as mean \pm SEM. For $n=8,{ }^{*} P<0.05$, significantly different from control group $(\mathrm{CG})$ rats; ${ }^{* *} P<0.05$, significantly different from stressed group 1 (SG1) rats; and ${ }^{* * *} P<0.05$, significantly different from SG2 rats. One-way ANOVA and Fisher's least significant difference tests were used. 
than that on the stressed group ones. However, the influence of noise stress on GRR and SPR induced by noise stress were reversed by MT (Fig. 2).

The results of Guth injury sore showed that it was significantly higher in stressed groups than in control groups and experimental groups $(P<0.01$, Fig. 3). Under a microscope, our pathological observation showed that there was gastric mucosa damage in stressed groups. Meanwhile, it is observed that gastric mucosa discontinuation, gland disorganization and remote hemorrhage on the surface as well as interstitial edema with inflammatory cell infiltration (Fig. 4). Due to the MT treatment, the damage score of experimental group rats was apparently reduced ( $P<0.05$, Fig. 3 ), and the pathological damages of mucosa were obviously palliated (Fig. 4).

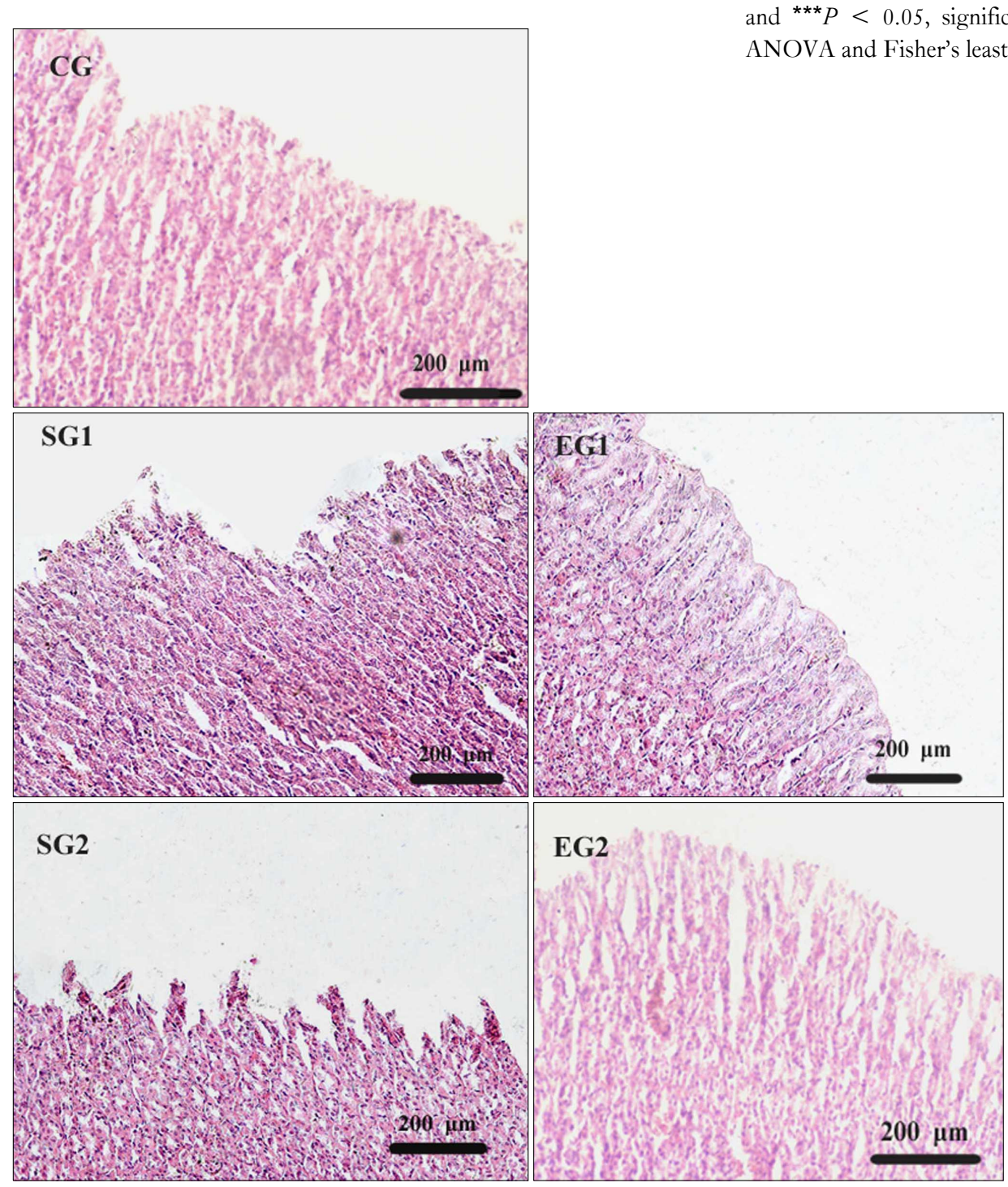

Figure 4. Pathological examination of gastric mucosa in rats $(\mathrm{H} \& \mathrm{E}$ stain, $\times 10)$.

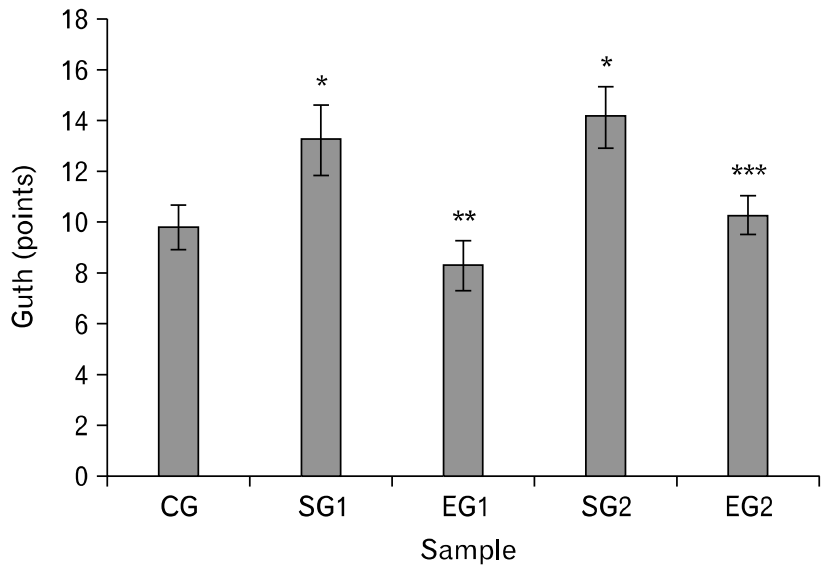

Figure 3. Effect of noise stress on Guth injury sore in rats and the reverse effect of melatonin. Results are shown as mean \pm SEM. For $n$ $=8,{ }^{*} P<0.05$, significantly different from control group (CG) rats; ${ }^{* *} P<0.05$, significantly different from stressed group 1 (SG1) rats; and ${ }^{* * *} P<0.05$, significantly different from SG2 rats. One-way ANOVA and Fisher's least significant difference tests were used. 
A

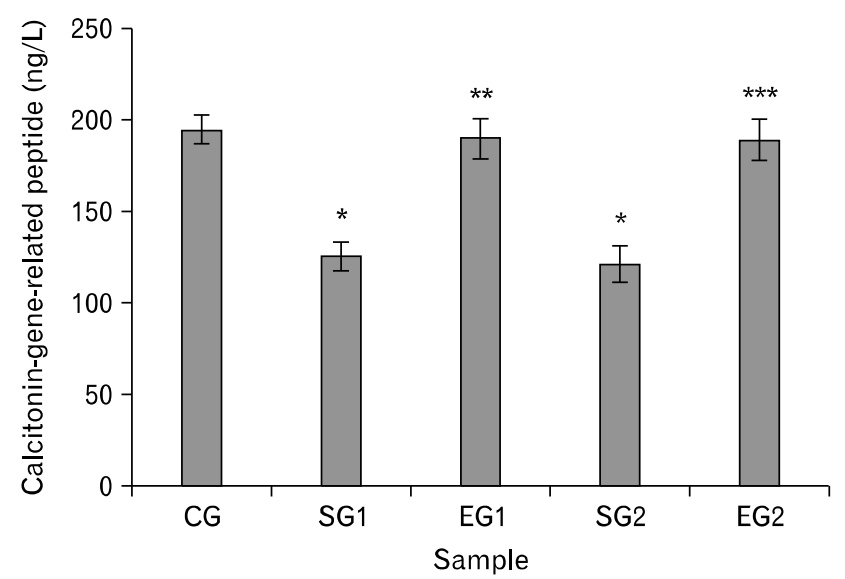

B

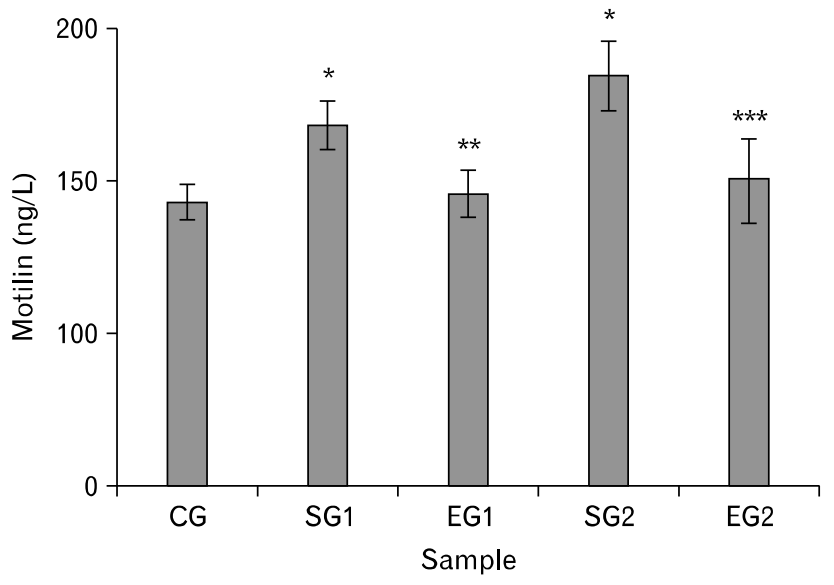

Figure 5. Effect of noise stress on calcitonin-gene-related peptide (A) and motilin (B) of blood plasma in rats and the reverse effect of melatonin. Results are shown as mean \pm SEM. For $n=8,{ }^{*} P<0.05$, significantly different from control group (CG) rats; ${ }^{* *} P<0.05$, significantly different from stressed group 1 (SG1) rats; and ${ }^{* *} P<0.05$, significantly different from SG2 rats. One-way ANOVA and Fisher's least significant difference tests were used.

A

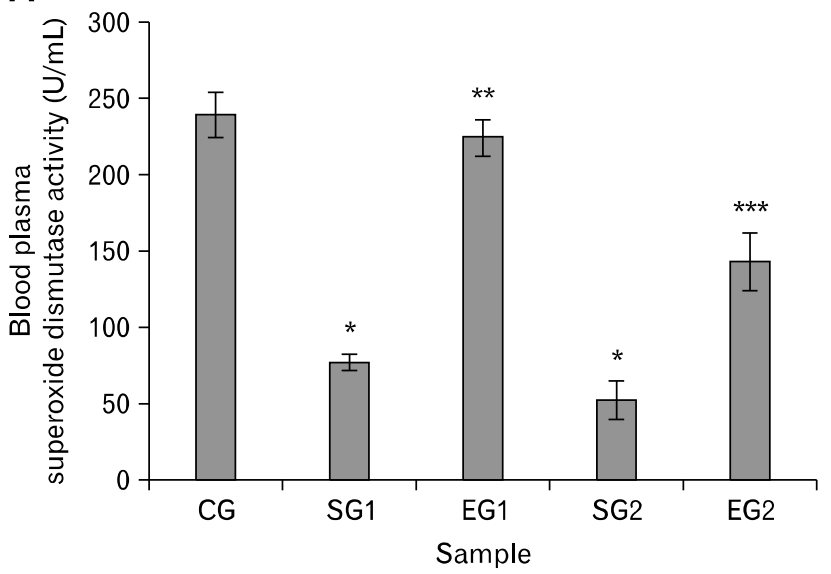

C

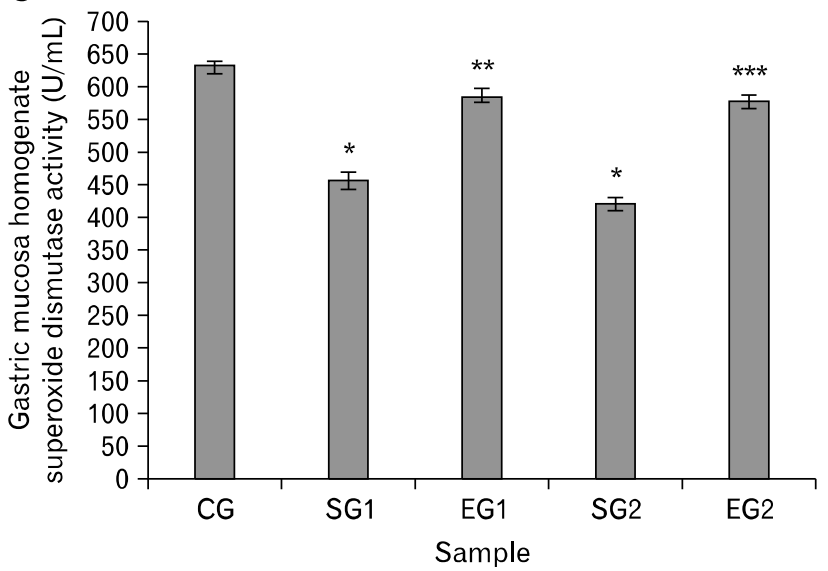

B

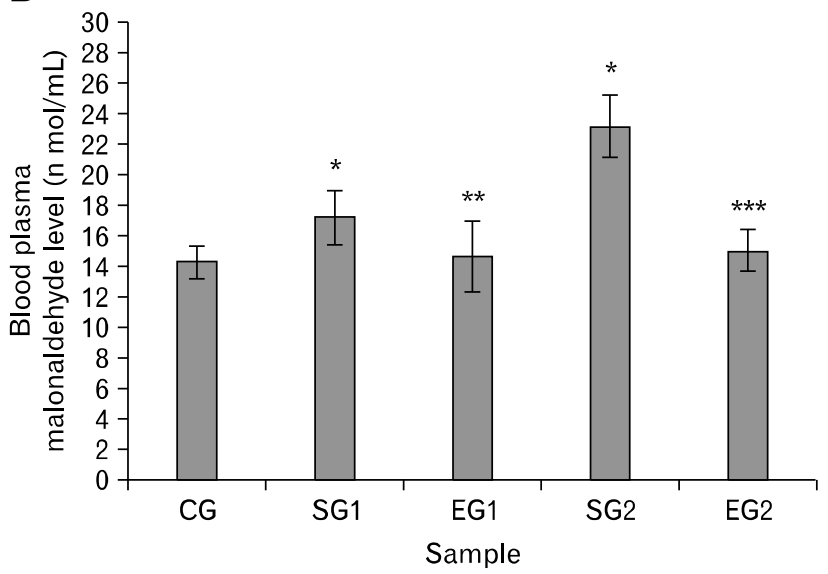

D

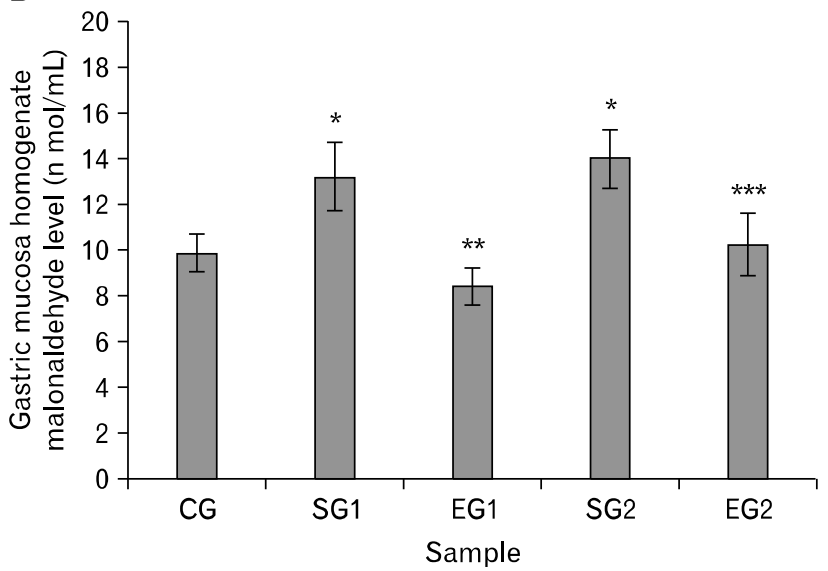

Figure 6. Effect of noise stress on superoxide dismutase activity (A and C) and malonaldehyde (B and D) of blood plasma (A and B) and gastric mucosa homogenate $(\mathrm{C}$ and $\mathrm{D})$ in rats and the reverse effect of melatonin. Results are shown as mean \pm SEM. For $\mathrm{n}=8$, ${ }^{*} P<0.05$, significantly different from control group $(\mathrm{CG})$ rats; ${ }^{* *} P<0.05$, significantly different from stressed group 1 (SG1) rats; and ${ }^{* \star *} P<0.05$, significantly different from SG2 rats. One-way ANOVA and Fisher's least significant difference tests were used. 
The levels of GI hormones in plasma are shown in Figure 5. To the rats exposed to noise stress, the CGRP level was decreased $(P<0.05$, Fig. 5A) whereas the motilin level was increased $(P<0.05$, Fig. 5B). Moreover, there were no differences between experimental group and control group rats in terms of motilin and CGRP levels ( $P>0.05$, Fig. 5).

Quantity of lipid peroxidation was used to represent oxidative stress, which was measured by the thiobarbituric acid colorimetric assay of malondialdehyde level and superoxide dismutase activity. For both blood plasma and gastric mucous homogenate, the superoxide dismutase activity was lower in stressed groups than that in control and experimental groups $(P<0.05$, Fig. 6A and $6 \mathrm{C})$; whereas the malondialdehyde level was higher in stressed groups than that in control and experimental groups $(P$ $<0.05$, Fig. $6 \mathrm{~B}$ and $6 \mathrm{D})$.

\section{Discussion}

In the present study, noise stress is confirmed to induce GIMD and gastric stress ulcer due to oxidative stress and activation of HPA axis which changes gut hormones. The results of present work show that MT plays a significant protective role in attenuating noise stress-induced GIMD and gastric stress ulcer. In addition, MT suppresses HPA axis activation and reverses gut hormones (motilin and CGRP) as well as oxidative stress markers (superoxide dismutase activity and malondialdehyde level).

Noise is one of important factors which induce human body stress and many non-auditory effects on GI tract. ${ }^{25,26}$ In general terms, these non-auditory responses tend to increase the level of alertness and activate HPA axis. These reflexes include a focused posture directed to the source of noise, a shunting blood away from vegetative regions like the stomach, a rise in adrenalin, noradrenalin and 17-hydroxycorticosteroids, and a release of glucose into the blood stream. ${ }^{27,28}$ Corticotropin-releasing factor (CRF) is a major signaling molecule released by hypothalamus into the portal circulation. Both of $\mathrm{CRF}$ and glucocorticoid receptor are found to localize in the cochlea. In addition, CRF expression has been described in the cochlea, indicating that not only the start point (CRF) but also the end point (glucocorticoid receptors) of the systemic HPA axis signaling are expressed in the cochlea. ${ }^{29} \mathrm{It}$ is believed that cochlea is an independent neuroendocrine organ. Besides other extra-hypothalamic that impacts within the brain, these hormones increase the activity of the HPA-axis and the release of corticosterone (eg, cortisol) from the adrenal cortex. ${ }^{30}$ Therefore, noise may affect the HPA-axis through the auditory system and result in the increased cortisol, ${ }^{31}$ which has been also confirmed in our study. Our results show that the level of cortisol in the plasma of stressed groups is higher than that of control group under noise stress. That maybe due to activate HPA axis and stimulate cochlea.

The secretion of MT with circadian rhythm in the blood of mammals is functionally linked to the adjustment of 24-hour cycles and to the circannual rhythm regulation. ${ }^{32}$ And the circadian change of MT is opposite to that of HPA-related hormones, suggesting maybe a connection between these two factors. It is reported that MT could reverse the HPA-axis activation induced by different stress which is similar to our results. ${ }^{33}$ However, our results also indicate that cortisol of blood plasma in experimental groups is different from that in control group, indicating the limitation of MT effect.

A growing number of studies have shown that stress could induce GIMD and gastric stress ulcer. For experimental animals, accumulating evidences have demonstrated that the most consistent patterns of GI motility alternations induced by various stresses (eg, restraint stress, foot shock stress, cold stress, and immersion stress) including delayed gastric emptying ${ }^{32,34,35}$ and accelerated colon transit. ${ }^{36,37}$ In the present study, noise stress inhibits gastric emptying of rats, which is supported by previous research. ${ }^{32,34,35}$ However, there are some controverts about small intestine motility under stress in previous studies. Some reports show that stress restrains small intestine motility, ${ }^{38,39}$ whereas the others suggest an accelerating effect. ${ }^{40,41}$ This may be attributed to the differences in the type of motility tested, complexity of the tasks, manner of stress induction, and the experimental protocols. We find that the noise stress accelerates food march in small intestine. Based on our results, the Guth injury score and pathology observation show that noise stress could produce gastric stress ulcer. In support of our findings, numerous reports indicate gastric stress ulcer is closely associated with stress including psychological stress ${ }^{42}$ and water immersion stress. ${ }^{43}$

The underlying mechanism of GIMD and gastric stress ulcer may be associated with abnormal excrete of gut hormones as a result of HPA axis activation. The present study reveals that noise stress increases motilin of but decreased CGRP of blood plasma. CGRP and motilin are known as key factors of gastric and duodenum ulcer. Besides regulating blood current and GI motility, CGRP maintains the homeostasis of intracellular $\mathrm{Ca}^{2+}$ and decreases the permeability of cell membrane to $\mathrm{Ca}^{2+}{ }^{44}$ Therefore, it can affect $\mathrm{Ca}^{2+}$ concentrations in and outside the cell, a key factor in the relaxation-contraction activities. In the 
previous study, gastric CGRP levels are found to decrease rapidly under water immersion restraint stress in both male and female mice. ${ }^{45}$ It is also suggested that both of mucosal expression of the CGRP gene and its serum levels are decreased with gastric damage in rat water immersion restraint stress model. ${ }^{46,47}$ It indicates that stress could down-regulate the level of CGRP, in both of serum and gastric mucosa which is similar to our results. The CGRP level in blood is a crucial regulating factor for gastric acid and ulcer development, and the exogenous CGRP can prevent lesion and accelerate lesion healing. ${ }^{48,49}$ Motilin can facilitate the intra-cellular calcium library to release $\mathrm{Ca}^{2+}$, which results in the contraction of smooth muscle, the acceleration of GI motility, the elevation of lower esophageal sphincter pressure and the contract cholecyst. ${ }^{50}$ Moreover, motilin participates in initiating the migrating motor complex (MMC) in the stomach, stimulates GI motility and accelerates gastric emptying. ${ }^{51}$ A previous study continuously monitored of fecal occult blood and GI motility and analyzes the plasma motilin concentration, GI endoscopy and gastric emptying in dogs. The results show that motilin is connected with ulcer and GI motility induced by nonsteroidal anti-inflammatory drugs. ${ }^{52}$ The motilin level in rats is also related to ulcerated antral mucosa tissue. $^{53}$

Enterochromaffin cells in GI tract could secrete MT and motilin. ${ }^{54}$ The finding that the concentration of MT in the GI tissues surpasses that in the blood by 10-100 times and in pineal gland by nearly 400 times suggests MT may also play an important role in digestive system. ${ }^{55}$ It has been shown that central nervous stimuli can increase duodenal bicarbonate secretion by release of mucosa $\mathrm{MT}^{56} \mathrm{MT}$ is reported to inhibit irregular spiking activity and reinforces the cyclic MMC pattern. ${ }^{57}$ These results suggest that MT affects GI motility in rats, which might be mediated by MT receptors in the GI tract, although central receptors for the hormone is possibly involved. ${ }^{58}$ Results of lots of articles suggest that CGRP plays an important role in the pathogenesis of gastric ulcer ${ }^{45,47,59}$ and GI motility disorder. ${ }^{60}$ Exogenous CGRP shows protective effect to reflux esophagitis ${ }^{61}$ and gastric mucosa ulcer. ${ }^{48,49}$ However, it is reported that GIMD is observed in patients with ulcers and those with non-ulcer dyspepsia compared with asymptomatic controls. ${ }^{62}$ That may indicate that gastric ulcer may influent GI motility itself. With the MT treatment, CGRP of blood plasma in experimental group rats was significantly different from that in stressed group rats. CGRP is involved in regulation of GIMD due to stress, no matter by affecting GI motility directly or through preventing gastric ulcer. These observations indicate that MT can reverse GIMD and gastric stress ulcer maybe through adjusting gut hormone secretion derangement induced by noise stress.

An important form of management for gastric ulcer diseases is to administer agents that strengthen the gastric mucosal barrier by reducing oxidative stress. Recent studies show that oxidative stress is involved in stress ulcer and GIMD in many animal stress models and peptic ulcer diseases of human beings. MT, the primary secretory product of the pineal gland, is a potent antioxidant and has been shown to be highly effective in reducing oxidative stress. In addition, MT or L-tryptophane accelerates ulcer healing with omeprazole treatment and this likely depends mainly upon the significant increments in plasma MT. ${ }^{63}$ Lipid peroxidation resulted from unsaturated fatty acids could induce the injury in biological membrane by oxygen free radicals. Malondialdehyde ${ }^{64}$ level and superoxide dismutase activity are used to estimate the oxidative stress in degrading and eliminating oxygen free radicals caused by lipid peroxidation ${ }^{65}$ that is also performed in the present study. It is well known that oxygen free radicals play an important role in gastric stress ulcer ${ }^{66}$ induced by different stress. In our study, noise stress leads to an increased lipid peroxidation of blood and gastric mucosa, as indicated by an increased malondialdehyde level of and a decreased superoxide dismutase activity.

MT is considered to be of a series of properties that would characterize an ideal free radical scavenger. It is believed that MT is present in all cells ${ }^{67}$ and concentration is in adequate amounts to protect tissues. Furthermore, MT is a broad-spectrum antioxidant by scavenging hydroxyl radical, alkoxyl radical, peroxyl radical, nitric oxide, and singlet oxygen. As an antioxidant, MT may be regenerated after radical quenching through different processes. ${ }^{68}$ It can also assist in stimulating antioxidant enzymes including superoxide dismutase, glutathione, peroxidase, and so on. ${ }^{69} \mathrm{MT}$ is also an indirect antioxidant ${ }^{70,71}$; metabolites of MT including N1-acetyl-N2-formyl-5-methoxykynuramine and N1acetyl-5-methoxykynuramine possess free radical-scavenging activities. ${ }^{72}$ After reaction between MT and a free radical, it yields an oxidized form of itself; its reactivity is so low that it is not toxic to cells. ${ }^{73}$ Many reports have shown that acute and chronic toxicity of MT is extremely low. ${ }^{74}$ Doses even up to one $\mathrm{g}$ daily by human volunteers revealed no negative side effects. ${ }^{75}$ There is widespread agreement that MT has minimal toxicity over a very wide dose range. Taken together, MT has most of characteristics of an ideal free radical scavenger.

However, molecular biology methods are not used in the present study that may provide further evidence for related mo- 
lecular mechanism. It is suggested that MT protects the gastric damage under diabetes through regulation of both matrix metalloproteinase- 1 and -13 which are mediated by activator protein-1 activation via extracellular signal-regulated kinase $1 / 2 .^{76} \mathrm{We}$ have focused on the MT as a free radical scavenger but the effect on the matrix metalloproteinase would also be of great interest for future studies.

In conclusion, the main finding of this study is that MT is a protective agent that prevents GIMD and gastric stress ulcer associated with noise stress. The possible mechanism may be attributed to its ability to reduce oxidative stress and attenuate the activation of HPA-axis as well as gut hormone disorder. Since MT has not been found to be of toxic activities in the body and its lethal dose 50 cannot be established in many animals, it may be relatively safe for clinical application.

\section{References}

1. Chrousos GP, Gold PW. The concepts of stress and stress system disorders. Overview of physical and behavioral homeostasis. JAMA 1992;267:1244-1252.

2. Maier SF, Watkins LR. Cytokines for psychologists: implications of bidirectional immune-to-brain communication for understanding behavior, mood, and cognition. Psychol Rev 1998;105:83-107.

3. Tomei F, Tomao E, Papaleo B, Baccolo TP, Alfi P. Study of some cardiovascular parameters after chronic exposure to noise. Int $\mathrm{J}$ Cardiol 1991;33:393-399.

4. Abey-Wickrama I, A'Brook MF, Gattoni FE, Herridge CF. Mentalhospital admissions and aircraft noise. Lancet 1969;2:1275-1277.

5. Ohrstrom E. Sleep disturbances caused by road traffic noise - Studies in laboratory and field. Noise Health 2000;2:71-78.

6. Belli S, Sani L, Scarficcia G, Sorrentino R. Arterial hypertension and noise: a cross-sectional study. Am J Ind Med 1984;6:59-65.

7. Castle JS, Xing JH, Warner MR, Korsten MA. Environmental noise alters gastric myoelectrical activity: Effect of age. World J Gastroenterol 2007;13:403-407.

8. Tomei F, Papaleo B, Baccolo TP, Persechino B, Spano G, Rosati MV. Noise and gastric secretion. Am J Ind Med 1994;26:367-372.

9. McIntosh LJ, Sapolsky RM. Glucocorticoids increase the accumulation of reactive oxygen species and enhance adriamycin-induced toxicity in neuronal culture. Exp Neurol 1996;141:201-206.

10. Owens MJ, Nemeroff CB. Physiology and pharmacology of corticotropin-releasing factor. Pharmacol Rev 1991;43:425-473.

11. Mu ZB, Huang YX, Zhao BM, Liu ZX, Zhang BH, Wang QL. Effect of explosive noise on gastrointestinal transit and plasma levels of polypeptide hormones. World J Gastroenterol 2006;12:2284-2287.

12. Velarde E, Alonso-Gomez AL, Azpeleta C, Isorna E, De Pedro N, Delgado MJ. Melatonin effects on gut motility are independent of the relaxation mediated by the nitrergic system in the goldfish. Comp Biochem Physiol A Mol Integr Physiol 2011;159:367-371.

13. Gitto E, Pellegrino S, Gitto P, Barberi I, Reiter RJ. Oxidative stress of the newborn in the pre- and postnatal period and the clinical utility of melatonin. J Pineal Res 2009;46:128-139.

14. Baydas G, Yasar A, Tuzcu M. Comparison of the impact of melatonin on chronic ethanol-induced learning and memory impairment between young and aged rats. J Pineal Res 2005;39:346-352.

15. Tan DX, Manchester LC, Sanchez-Barcelo E, Mediavilla MD, Reiter RJ. Significance of high levels of endogenous melatonin in Mammalian cerebrospinal fluid and in the central nervous system. Curr Neuropharmacol 2010;8:162-167.

16. Zhang L, Zhang HQ, Liang XY, Zhang HF, Zhang T, Liu FE. Melatonin ameliorates cognitive impairment induced by sleep deprivation in rats: role of oxidative stress, BDNF and CaMKII. Behav Brain Res 2013;256:72-81.

17. Ashby CR, Jr., Edwards E, Wang RY. Electrophysiological evidence for a functional interaction between 5-HT1A and 5-HT2A receptors in the rat medial prefrontal cortex: an iontophoretic study. Synapse 1994;17:173-181.

18. Kvetnoy IM, Ingel IE, Kvetnaia TV, et al. Gastrointestinal melatonin: cellular identification and biological role. Neuro Endocrinol Lett 2002;23:121-132.

19. Bubenik GA, Hacker RR, Brown GM, Bartos L. Melatonin concentrations in the luminal fluid, mucosa, and muscularis of the bovine and porcine gastrointestinal tract. J Pineal Res 1999;26:56-63.

20. Chen CQ, Fichna J, Bashashati M, Li YY, Storr M. Distribution, function and physiological role of melatonin in the lower gut. World J Gastroenterol 2011;17:3888-3898.

21. Thor PJ, Krolczyk G, Gil K, Zurowski D, Nowak L. Melatonin and serotonin effects on gastrointestinal motility. J Physiol Pharmacol 2007;58 Suppl 6:97-103.

22. Mittelstadt SW, Hemenway CL, Spruell RD. Effects of fasting on evaluation of gastrointestinal transit with charcoal meal. J Pharmacol Toxicol Methods 2005;52:154-158.

23. Dong DL, Wang QH, Chen W, et al. Contrasting effects of tetraethylammonium and 4-aminopyridine on the gastrointestinal function of mice. Eur J Pharmacol 2005;509:179-185.

24. Guth PH, Aures D, Paulsen G. Topical aspirin plus $\mathrm{HCl}$ gastric lesions in the rat. Cytoprotective effect of prostaglandin, cimetidine, and probanthine. Gastroenterology 1979;76:88-93.

25. Yates DA, Santos J, Soderholm JD, Perdue MH. Adaptation of stress-induced mucosal pathophysiology in rat colon involves opioid pathways. Am J Physiol Gastrointest Liver Physiol 2001;281:G124G128.

26. Beglinger $\mathrm{C}$, Degen L. Role of thyrotrophin releasing hormone and corticotrophin releasing factor in stress related alterations of gastrointestinal motor function. Gut 2002;(51 suppl 1):i45-i49.

27. Arguelles AE, Ibeas D, Ottone JP, Chekherdemian M. Pituitaryadrenal stimulation by sound of different frequencies. J Clin Endocrinol Metab 1962;22:846-852.

28. Michalak R, Ising H, Rebentisch E. Acute circulatory effects of military low-altitude flight noise. Int Arch Occup Environ Health 1990;62:365-372.

29. Basappa J, Graham CE, Turcan S, Vetter DE. The cochlea as an independent neuroendocrine organ: expression and possible roles of a local hypothalamic-pituitary-adrenal axis-equivalent signaling system. Hear Res 2012;288:3-18.

30. Masini CV, Babb JA, Nyhuis TJ, Day HE, Campeau S. Auditory cortex lesions do not disrupt habituation of HPA axis responses to re- 
peated noise stress. Brain Res 2012;1443:18-26.

31. Abe H, Hidaka N, Kawagoe C, et al. Prenatal psychological stress causes higher emotionality, depression-like behavior, and elevated activity in the hypothalamo-pituitary-adrenal axis. Neurosci Res 2007;59:145-151.

32. Reiter RJ. The melatonin rhythm: both a clock and a calendar. Experientia 1993;49:654-664.

33. Saito S, Tachibana T, Choi YH, Denbow DM, Furuse M. ICV melatonin reduces acute stress responses in neonatal chicks. Behav Brain Res 2005;165:197-203.

34. Ochi M, Tominaga K, Tanaka F, et al. Effect of chronic stress on gastric emptying and plasma ghrelin levels in rats. Life Sci 2008;82: 862-868.

35. Iwa M, Nakade Y, Pappas TN, Takahashi T. Electroacupuncture elicits dual effects: stimulation of delayed gastric emptying and inhibition of accelerated colonic transit induced by restraint stress in rats. Dig Dis Sci 2006;51:1493-1500.

36. Miampamba M, Million M, Yuan PQ, Larauche M, Tache Y. Water avoidance stress activates colonic myenteric neurons in female rats. Neuroreport 2007;18:679-682.

37. Nakade Y, Fukuda H, Iwa M, et al. Restraint stress stimulates colonic motility via central corticotropin-releasing factor and peripheral 5- $\mathrm{HT}_{3}$ receptors in conscious rats. Am J Physiol Gastrointest Liver Physiol 2007;292:G1037-1044.

38. Tsukada F, Saitoh M, Ohkubo Y. Development of an experimental system for evaluation of the effect of stress on intestinal motility using a radionuclide, ${ }^{51} \mathrm{Cr}$, in the rat. Biol Pharm Bull 2000;23:1384-1386.

39. Cao SG, Wu WC, Han Z, Wang MY. Effects of psychological stress on small intestinal motility and expression of cholecystokinin and vasoactive intestinal polypeptide in plasma and small intestine in mice. World J Gastroenterol 2005;11:737-740.

40. Muelas MS, Ramirez P, Parrilla P, et al. Vagal system involvement in changes in small bowel motility during restraint stress: an experimental study in the dog. Br J Surg 1993;80:479-483.

41. Muelas MS, Ramirez P, Parrilla P, et al. Role of the vagal system in changes in small intestinal motility induced by psychological stress. Experimental study on dogs. Rev Esp Enferm Dig 1992;81:383-387.

42. Levenstein S, Rosenstock S, Jacobsen RK, Jorgensen T. Psychological stress increases risk for peptic ulcer, regardless of Helicobacter pylori infection or use of non-steroidal anti-inflammatory drugs. Clin Gastroenterol Hepatol 2015;13:498-506, e1.

43. Zheng YF, Xie JH, Xu YF, et al. Gastroprotective effect and mechanism of patchouli alcohol against ethanol, indomethacin and stressinduced ulcer in rats. Chem Biol Interact 2014;222C:27-36.

44. Cullen JJ, Ephgrave KS, Caropreso DK. Gastrointestinal myoelectric activity during endotoxemia. Am J Surg 1996;171:596-599.

45. Shimozawa N, Okajima K, Harada N, et al. Contribution of sensory neurons to sex difference in the development of stress-induced gastric mucosal injury in mice. Gastroenterology 2006;131:1826-1834.

46. Kang JM, Kim N, Kim B, et al. Gastroprotective action of Cochinchina momordica seed extract is mediated by activation of CGRP and inhibition of cPLA(2)/5-LOX pathway. Dig Dis Sci 2009;54: 2549-2560.

47. Szlachcic A, Sliwowski Z, Krzysiek-Maczka G, et al. New satiety hormone nesfatin-1 protects gastric mucosa against stress-induced injury: mechanistic roles of prostaglandins, nitric oxide, sensory nerves and vanilloid receptors. Peptides 2013;49:9-20.

48. Ohno T, Hattori Y, Komine R, et al. Roles of calcitonin gene-related peptide in maintenance of gastric mucosal integrity and in enhancement of ulcer healing and angiogenesis. Gastroenterology 2008;134: 215-225.

49. Li NS, Luo XJ, Dai Z, et al. Beneficial effects of capsiate on ethanol-induced mucosal injury in rats are related to stimulation of calcitonin gene-related Peptide release. Planta Med 2012;78:24-30.

50. Poitras P, Peeters TL. Motilin. Curr Opin Endocrinol Diabetes Obes 2008;15:54-57.

51. Chen CY, Tsai CY. Ghrelin and motilin in the gastrointestinal system. Curr Pharm Des 2012;18:4755-4765.

52. Narita T, Okabe N, Hane M, et al. Nonsteroidal anti-inflammatory drugs induce hypermotilinemia and disturbance of interdigestive migrating contractions in instrumented dogs. J Vet Pharmacol Ther 2006;29:569-577.

53. Sun FP, Song YG. Effect of environmental hyperthermia on gastrin, somatostatin and motilin in rat ulcerated antral mucosa. World J Gastroenterol 2004;10:3528-3530.

54. Bubenik GA. Localization, physiological significance and possible clinical implication of gastrointestinal melatonin. Biol Signals Recept 2001;10:350-366.

55. Lee PP, Pang SF. Melatonin and its receptors in the gastrointestinal tract. Biol Signals 1993;2:181-193.

56. Sjoblom M, Flemstrom G. Central nervous stimuli increase duodenal bicarbonate secretion by release of mucosal melatonin. J Physiol Pharmacol 2001;52:671-678.

57. Merle A, Delagrange $P$, Renard $P$, et al. Effect of melatonin on motility pattern of small intestine in rats and its inhibition by melatonin receptor antagonist S 22153. J Pineal Res 2000;29:116-124.

58. Drago F, Macauda S, Salehi S. Small doses of melatonin increase intestinal motility in rats. Dig Dis Sci 2002;47:1969-1974.

59. Luo XJ, Li NS, Zhang YS, et al. Vanillyl nonanoate protects rat gastric mucosa from ethanol-induced injury through a mechanism involving calcitonin gene-related peptide. Eur J Pharmacol 2011;666: 211-217.

60. Smith AS, Smid SD. Impaired capsaicin and neurokinin-evoked colonic motility in inflammatory bowel disease. J Gastroenterol Hepatol 2005;20:697-704.

61. Konturek PC, Brzozowska I, Targosz A, et al. Esophagoprotection mediated by exogenous and endogenous melatonin in an experimental model of reflux esophagitis. J Pineal Res 2013;55:46-57.

62. Stanghellini V, Ghidini C, Maccarini MR, Paparo GF, Corinaldesi R, Barbara L. Fasting and postprandial gastrointestinal motility in ulcer and non-ulcer dyspepsia. Gut 1992;33:184-190.

63. Celinski K, Konturek SJ, Konturek PC, et al. Melatonin or L-tryptophan accelerates healing of gastroduodenal ulcers in patients treated with omeprazole. J Pineal Res 2011;50:389-394.

64. Nielsen F, Mikkelsen BB, Nielsen JB, Andersen HR, Grandjean P. Plasma malondialdehyde as biomarker for oxidative stress: reference interval and effects of life-style factors. Clin Chem 1997;43:1209-1214.

65. Zablocka A, Janusz M. The two faces of reactive oxygen species. Postepy Hig Med Dosw (Online) 2008;62:118-124.

66. Farias-Silva E, Cola M, Calvo TR, et al. Antioxidant activity of indigo and its preventive effect against ethanol-induced DNA damage in rat gastric mucosa. Planta Med 2007;73:1241-1246. 
67. Reiter RJ, Tan DX, Rosales-Corral S, Manchester LC. The universal nature, unequal distribution and antioxidant functions of melatonin and its derivatives. Mini Rev Med Chem 2013;13:373-384.

68. Blanchard-Fillion B, Servy C, Ducrocq C. 1-Nitrosomelatonin is a spontaneous NO-releasing compound. Free Radic Res 2001;35: 857-866.

69. Rodriguez C, Mayo JC, Sainz RM, et al. Regulation of antioxidant enzymes: a significant role for melatonin. J Pineal Res 2004;36:1-9.

70. Baydas G, Ozveren F, Akdemir I, Tuzcu M, Yasar A. Learning and memory deficits in rats induced by chronic thinner exposure are reversed by melatonin. J Pineal Res 2005;39:50-56.

71. Allegra M, Reiter RJ, Tan DX, Gentile C, Tesoriere L, Livrea MA. The chemistry of melatonin's interaction with reactive species. J Pineal Res 2003;34:1-10.
72. Galano A, Tan DX, Reiter RJ. On the free radical scavenging activities of melatonin's metabolites, AFMK and AMK. J Pineal Res 2013;54:245-257.

73. Lewis AJ, Kerenyi NA, Feuer G. Neuropharmacology of pineal secretions. Drug Metabol Drug Interact 1990;8:247-312.

74. Vijayalaxmi, Meltz ML, Reiter RJ, Herman TS. Melatonin and protection from genetic damage in blood and bone marrow: wholebody irradiation studies in mice. J Pineal Res 1999;27:221-225.

75. Nordlund JJ, Lerner AB. The effects of oral melatonin on skin color and on the release of pituitary hormones. J Clin Endocrinol Metab 1977;45:768-774.

76. Pradeepkumar Singh L, Vivek Sharma A, Swarnakar S. Upregulation of collagenase- 1 and -3 in indomethacin-induced gastric ulcer in diabetic rats: role of melatonin. J Pineal Res 2011;51:61-74. 